\title{
Concurrent production and relative quantification of vasicinone from in vivo and in vitro plant parts of Malabar nut (Adhatoda vasica Nees)
}

\author{
Jitendriya Panigrahi ${ }^{1}$ Saikat Gantait ${ }^{2,3}$ (D) Illa C. Patel $^{4}$
}

Received: 3 June 2017/ Accepted: 10 July 2017/Published online: 16 August 2017

(c) The Author(s) 2017. This article is an open access publication

\begin{abstract}
The present study documents a simultaneous production and comparative assessment of extracted vasicinone from in vivo (leaves and stems) and in vitro (leaves, stems and calli) plant parts of Adhatoda vasica Nees, a well-known medicinal plant. High-performance thin layer chromatography (HPTLC) analysis of the above-mentioned plant parts, collected at their 60-day-old growth stage, was performed via methanolic extraction and with the aid of toluene:butanol:butyl acetate $(9: 0.5: 0.5 ; v / v / v)$ solvent system. The method was validated with the help of aluminium sheet precoated with silica gel $60 \mathrm{~F}_{254}$ TLC plates, following the ICH guidelines in order to maintain accuracy, precision and repeatability. Correlation coefficient, limit of detection and limit of quantification values were found to be reasonable. The outcome revealed a linearity that ranged between 2 and $6 \mu \mathrm{g} / \mathrm{spot}$. During the comparison of estimated vasicinone quantity from in vivo and in vitro plant
\end{abstract}

Saikat Gantait

saikatgantait@yahoo.com

Jitendriya Panigrahi

jitenp2001@gmail.com

Illa C. Patel

illa.botany@gmail.com

1 Department of Biotechnology, Shri A. N. Patel P. G. Institute, Anand, Gujarat 388001, India

2 All India Coordinated Research Project on Groundnut, Directorate of Research, Bidhan Chandra Krishi Viswavidyalaya, Kalyani, Nadia, West Bengal 741235, India

3 Department of Genetics and Plant Breeding, Faculty of Agriculture, Bidhan Chandra Krishi Viswavidyalaya, Mohanpur, Nadia, West Bengal 741252, India

4 Department of Life Sciences, Hemchandracharya North Gujarat University, Patan, Gujarat 384265, India parts, it was evident that in vitro samples produced relatively higher vasicinone than that of the in vivo counterparts. Maximum vasicinone $(6.402 \pm 0.010 \%$ of dry weight) production was quantified from in vitro leaves followed by calli $(5.222 \pm 0.092 \%$ of dry weight $)$ and in vitro stems $(2.007 \pm 0.041 \%$ of dry weight). On the other hand, in vivo leaves and stems produced comparatively lower quantities of vasicinone $(2.412 \pm 0.139$ and $1.933 \pm 0.046 \%$ of dry weight, respectively) suggesting the in vitro clonal propagation as a superior approach in comparison to in vivo propagation. Nonetheless, simultaneous production from both the sources (in vivo and in vitro plant parts) provides a new avenue for augmented production of vasicinone.

Keywords Adhatoda vasica . HPTLC - Malabar nut . Medicinal plant · Vasicinone

\section{Introduction}

Adhatoda vasica Nees (syn, Justicia adhatoda L.), commonly known as Malabar Nut (or Vasaka) is a perennial shrub and belongs to the family Acanthaceae. It grows in sub-Himalayan tracts and has conventionally been utilized in Ayurvedic and Unani medicine for more than 2000 years (Jayapaul et al. 2005). It is basically an evergreen shrub of 1-2.5 m height with opposite ascending branches producing a vile smell and bitter taste. Several ethnopharmacological studies on A. vasica reported the aerial portions of the plant (like stem, leaf, flower, fruit and seeds) to contain vasicine, vasicinone (Suthar et al. 2009), vasicine acetate and 2-acetyl benzyl amine (Ignacimuthus and Shanmugam 2010), adhatodine and vasicoline (Ahmad et al. 2009). A. vasica leaves and stems have been utilized 
as a herbal medication for allergen-induced respiratory diseases, rheumatism, malarial fever, gastrointestinal disorders, haemorrhage, skin diseases and many other ailments, occurring in countries like India, Nepal, Pakistan and Sri Lanka (reviewed by Claeson et al. 2000). A. vasica has been included in the WHO manual for health workers in Southeast Asia owing to its recurrent usage, conveniently devoid of any serious side effects (World Health Organization 1990). Vasicinone, the central autooxidation by-product of vasicine, has been documented to exhibit bronchodilatory, cardiac-stimulating and anti-anaphylactic effects (Bhide et al. 1976; Shinawie 2002). One of the commercial produces of A. vasica is Adulsa, a cough syrup (Roja et al. 2011). There is a significant requirement of $A$. vasica-based drugs in the Indian subcontinent and this demand is being satisfied from the natural plant population so far. Inaptly, seed germination rate of A. vasica is poor and traditional propagation through vegetative approach is inadequate (Mathew et al. 1998), which prompts significant depletion of the source of pharmaceutically important metabolites like vasicinone. This existing scenario calls for an alternative approach in the form of in vitro propagation that could complement the traditional system of propagation, and crop up as a better source for secondary metabolites extraction. Nowadays plant organ, tissue and cell culture system have been exhaustively explored as a substitute for the production of secondary metabolites, avoiding any loss in natural plant population in majority of the medicinal plant species (Ramachandra Rao and Ravishankar 2002; Komaraiah et al. 2003; Gantait et al. 2011). Yet, the comparative assessment of secondary metabolite among in vitro and in vivo plant parts is of utmost importance from an economic viewpoint; however, such study has been attempted only once (Garg et al. 2016) in A. vasica, to the best of our knowledge. The propagation conditions (in vitro or in vivo) assisting the accumulation of high concentration of vasicinone in A. vasica are undetermined as well.

Earlier reported techniques for the vasicinone quantification comprises high-performance capillary electrophoresis (HPCE) (Avula et al. 2008), reversed-phase high-performance liquid chromatography (RPHPLC) (Garg et al. 2016), and ultra-high-performance liquid chromatography coupled with triple quadrupole linear ion trap mass spectrometry (UHPLC-ESI/MS/MS) (Singh et al. 2016), along with high-performance thin layer chromatography (HPTLC) as well. Presently, HPTLC is being widely used for its systematic method validation, consistency in the quantification of analytes (precisely in nanogram levels) and cost effectiveness. The prime benefit of HPTLC is curtailment of time and cost per analysis (Dharmender et al. 2010). Effective use of HPTLC has been well documented in some major economically important medicinal plants for their respective secondary metabolite assay (Gantait et al. 2011; Swain et al. 2012; Varghese et al. 2013; Bala et al. 2015; Kumar et al. 2016; Shuayprom et al. 2016), but only three reports are available on vasicinone quantification from in vivo plant samples of A. vasica (Das et al. 2005; Suthar et al. 2009; Roja et al. 2011) with none on comparative quantification (between in vivo and in vitro plant parts) of vasicinone.

The present study was therefore focused on the simultaneous production and relative assessment of vasicinone through HPTLC from both the in vivo and in vitro plant parts of A. vasica, and optimizing the major sink of accumulation.

\section{Materials and methods}

\section{Collection of plant materials}

The stem-cuttings of A. vasica Nees were collected from Medicinal and Aromatic Research Centre, Anand Agriculture University, Anand, Gujarat, India, and propagated through traditional vegetative method (Fig. 1a). The freshly developed and fully opened 60-day-old leaves along with tender stems of similar growth stage were harvested to measure the vasicinone content.

The in vivo-collected nodal-segment explants were inoculated in Murashige and Skoog (1962) (MS) medium fortified with $1.1 \mathrm{mg} / \mathrm{l}$ of $N^{6}$-benzyladenine for in vitro initiation and multiplication of axillary shoots following the protocol of Panigrahi and Patel (2014). Simultaneously, for in vitro callus induction, the in vivo-collected nodal segments were inoculated in MS medium fortified with $1 \mathrm{mg} / \mathrm{l}$ of 2,4-dichlorophenoxy acetic acid. Leaves and stems of in vivo plants as well as in vitro-regenerated leaves, stems (Fig. 1b) and induced calli (Fig. 1c) were used for HPTLC analysis considering vasicinone as standard.

\section{Preparation of sample solution}

To develop the appropriate fingerprinting along with effective extract preparation, sample solutions were evaluated. The in vivo and in vitro samples (weighing $1 \mathrm{~g}$ of the oven dried and powdered) were extracted using methanol $(50 \mathrm{ml})$ under reflux in a thimble with extractor volume 200 and 300-ml flask volume of Soxhlet apparatus (Borosil $^{\circledR}$, Mumbai, India). The extracts were filtered across Whatman filter paper (no. 1) and concentrated under vacuum and subsequently the volume was adjusted with methanol to develop a concentration of $1 \mathrm{mg} / \mathrm{ml}$ that were used for the chromatography analysis. One milligramme standard of vasicinone (purity: 97\%, Natural Remedies Pvt. 

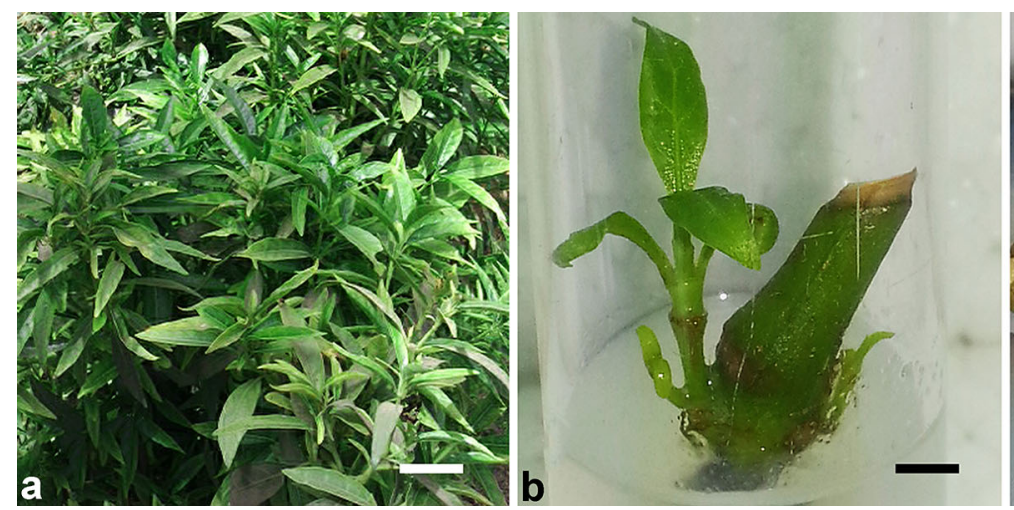

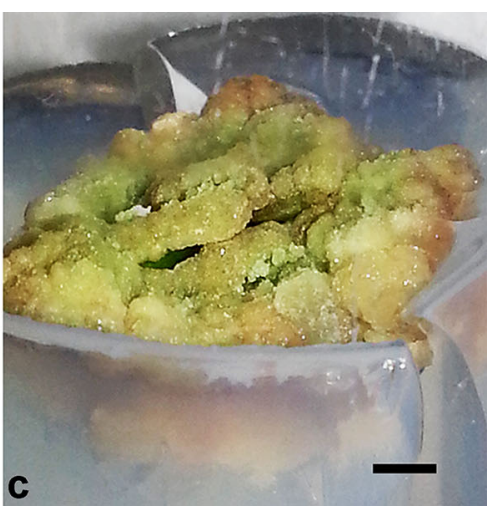

and leaf regeneration from nodal-segment explant; $\mathbf{c}$ in vitro callus induction from nodal-segment explant simultaneous production, extraction and quantification of vasicinone. a In vivo propagation through stem-cuttings; $\mathbf{b}$ in vitro multiple shoot

Ltd, Bengaluru, India) was dissolved in $1 \mathrm{ml}$ of methanol. It was further diluted to get the concentration of $0.01 \mathrm{mg} /$ ml. Toluene:butanol:butyl acetate $(9: 0.5: 0.5 ; v / v / v)$ was used for co-chromatography with the different extracted samples.

\section{Chromatographic analysis}

Five-microlitre solutions from each sample were spotted in the form of bands by $100-\mu 1$ syringe (from Hamilton ${ }^{\circledR}$, Sigma-Aldrich, USA) in a CAMAG Linomat 5 Automatic Sample Spotter (Camag Muttenz, Switzerland) on an aluminium sheet precoated with silica gel $60 \mathrm{~F}_{254}$ TLC plates $(20 \times 10 \mathrm{~cm}$ with $0.25 \mathrm{~mm}$ thickness) (E. Merck, Germany). After drying, the plate was developed in toluene:butanol:butyl acetate $(9: 0.5: 0.5 ; \quad v / v / v)$ solvent system to a distance of $8 \mathrm{~cm}$ and scanned at $233 \mathrm{~nm}$. The peak areas were measured and the quantity of vasicinone was estimated using the calibration curve with the assistance of winCATS Planar Chromatography Manager software. The whole procedure was carried out at $25 \pm 2{ }^{\circ} \mathrm{C}$ with a relative humidity of $40 \%$ while the analytical grade chemicals used in the experiment were purchased from Loba chemicals, Mumbai, India.

\section{Method validation}

The HPTLC method for quantification of vasicinone was validated for the following parameters-linearity, calibration curve, instrumental precision and repeatability. Calibration curve was drawn up and tested for linearity as well as validation of the suggested method. The coefficient of variance $(\mathrm{CV} \%)$, correlation coefficient $(r)$, and the linearity of results were analysed. Calibration curve of each sample was obtained by plotting peak areas vs concentrations of applied samples. The instrumental precision and the repeatability of the scanning technique was confirmed by repetitive scanning $(n=9)$ of the same spot (on TLC plate) of each sample and expressed as relative standard deviation (\%RSD).

Sensitivity The sensitivity of the proposed method was deduced against limit of detection (LOD) and limit of quantification (LOQ). The LOD and LOQ were calculated based on the gradient of calibration (S) curve and standard deviation (sdv) of the blank sample following the formula proposed by Alam et al. (2011).

Specificity It was established by evaluating standard vasicinone, test samples and diluent. The bands for vasicinone from test sample solutions were affirmed via correlating the $R_{\mathrm{f}}$ and spectra of the bands to those of the standard vasicinone. Analysis of peak purity of all the compounds was performed analogizing the spectra at start, middle, and end positions of the bands. The $R_{\mathrm{f}}$ values and colour of the resolved bands were noted.

\section{Data analysis}

The HPTLC analysis for the quantitative determination of vasicinone on different in vivo and in vitro plant samples was replicated thrice and the recorded data were put to oneway analysis of variance. The significant variation among the vasicinone quantity (mean $\pm \mathrm{SE}$ ) was analysed by Duncan's multiple range test (Duncan 1955) at $P=0.05$ level with the aid of SPSS (Version 20, SPSS Inc. Chicago, USA) software package.

\section{Results and discussion}

Preparatory TLC studies showed that the toluene:butanol:butyl acetate $(9: 0.5: 0.5 ; v / v / v)$ solvent system was ideal as a mobile phase that produced $R_{\mathrm{f}}$ 0.4-0.09 for vasicinone and well-resolved spots were found from the in vivo and in vitro plant samples (Fig. 2). The spots were 


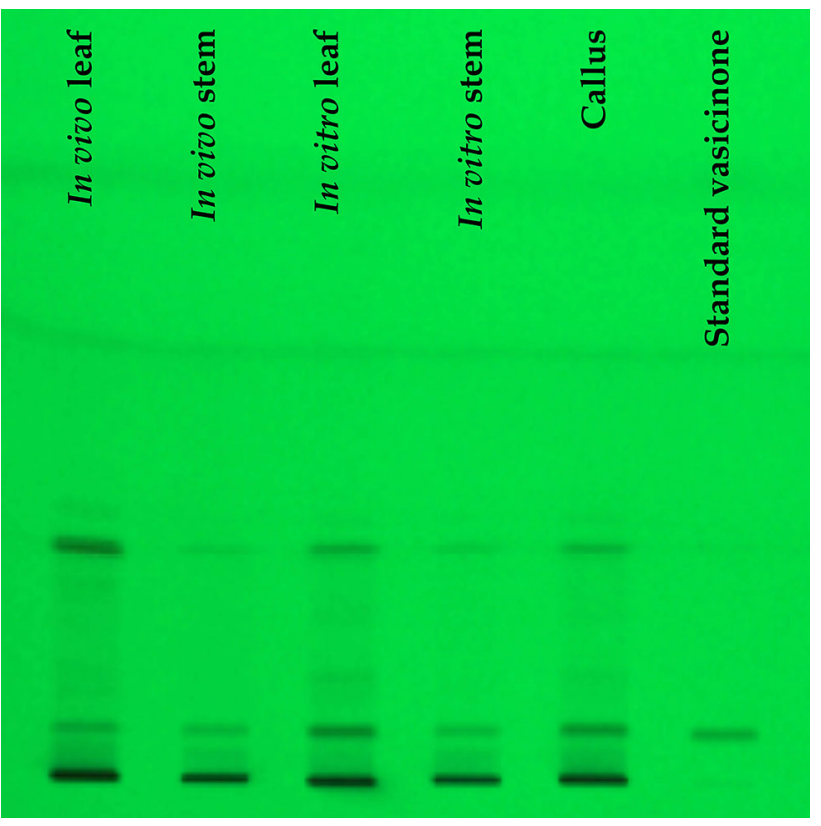

Fig. 2 TLC profile of the Adhatoda vasica Nees samples at $233 \mathrm{~nm}$ remission; samples extracted from in vivo and in vitro sources along with vasicinone as standard

observed at $233 \mathrm{~nm}$ and the resultant three-dimensional densitogram configurations of the test samples and standard vasicinone demonstrated that the peaks for all the samples corresponding to $R_{\mathrm{f}} 0.04$ were overlaying (Figs. 3, 4). The features of the corresponding spectrum of this peak were noted to match precisely with each other, revealing the compounds analogous to $R_{\mathrm{f}}$ of the standards and the in vivo and in vitro plant samples to be identical.

\section{Analytical method validation}

The linearity of the calibration curve was found between 2 and $6 \mu \mathrm{g} / \mathrm{spot}$ with a respective correlation coefficient ( $r$ value of 0.99 with standard deviation-sdv of 3.07), for vasicinone (Fig. 5). The regression equation for the calibration plot for vasicinone is $Y=1235+754.4 \times X$. Percentage $(w / w)$ of vasicinone was deduced using the peak area factor.

The densitometric method was found to be precise with RSD $20.93 \%$ (for intra-day) and $19.96 \%$ (for inter-day) by scanning the same spot of vasicinone simultaneously, with a \%CV value of 1.884 (intra-day) and 1.797 (inter-day), respectively. The repeatability of the method was investigated by evaluating nine applications of the same standard solution $\% \mathrm{CV}=1.884$ for intra-day and 1.797 for interday $(n=9)$. Validation data determines the precision of the methodology adapted in the current study in agreement with the $1 \mu \mathrm{g} / \mathrm{spot} \mathrm{LOD}$ and $3 \mu \mathrm{g} / \mathrm{spot} \mathrm{LOQ}$.

For the instruments precision, the specificity study was carried out taking vasicinone as standard, wherein test samples along with the mobile phase and diluent were taken on different tracks. The method proved to be specific for vasicinone since it resolved the compound $\left(R_{\mathrm{f}}=0.04\right)$ well in the presence of other components (Fig. 6).

\section{Determination and comparative analysis of vasicinone content from in vivo and in vitro plant samples}

The quantities (mean $\pm \mathrm{SE}$ ) of vasicinone procured from various in vivo and in vitro plant samples, expressed as \% of dry weight, are given in Fig. 7. It was evident from our study that vasicinone could be produced concurrently from different parts of the plant irrespective of in vitro or in vivo
Fig. 3 Overlay spectra of methanolic extract of different parts of in vivo and in vitro grown Adhatoda vasica Nees at $233 \mathrm{~nm}$

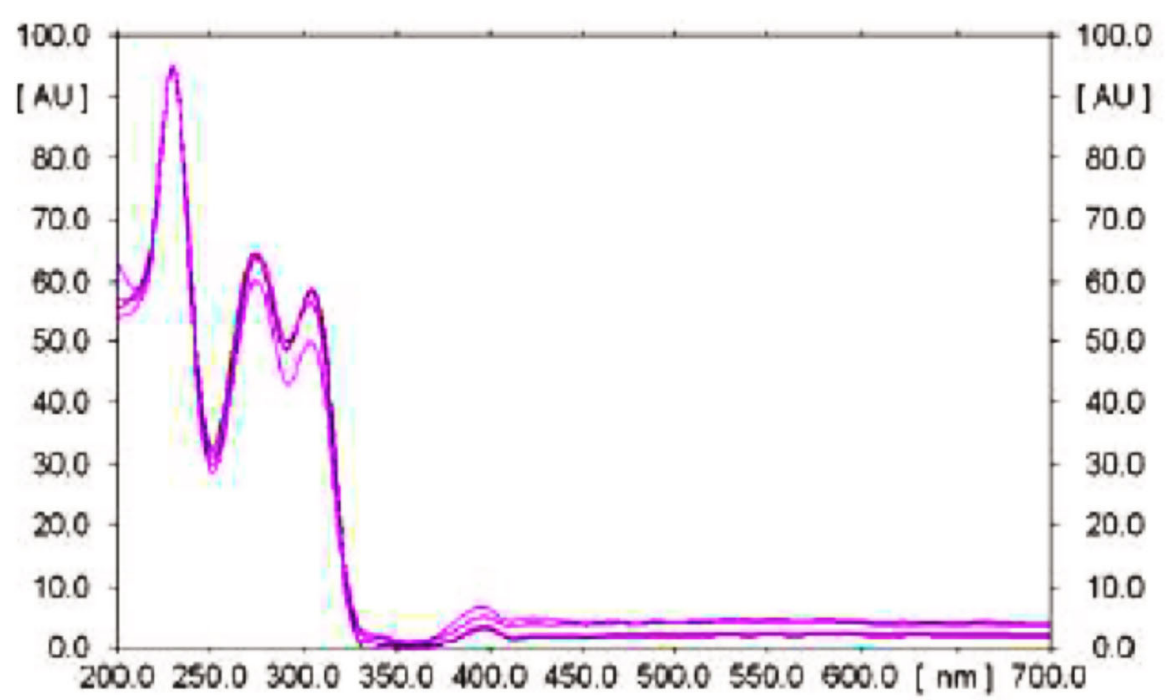




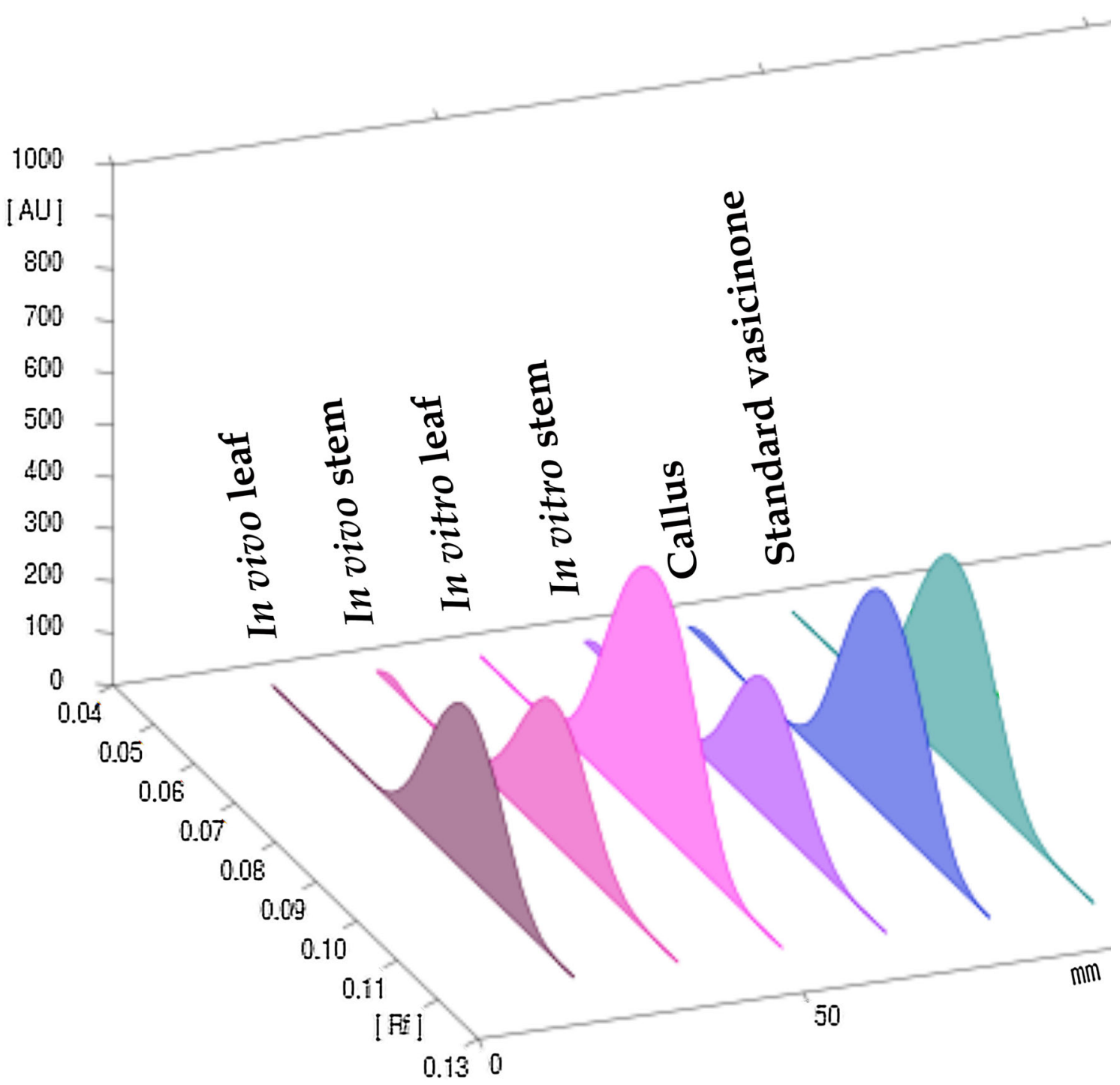

Fig. 4 HPTLC fingerprinting (all tracks at $233 \mathrm{~nm}$ ) of vasicinone along with the methanolic extract of different parts of in vivo and in vitro grown Adhatoda vasica Nees

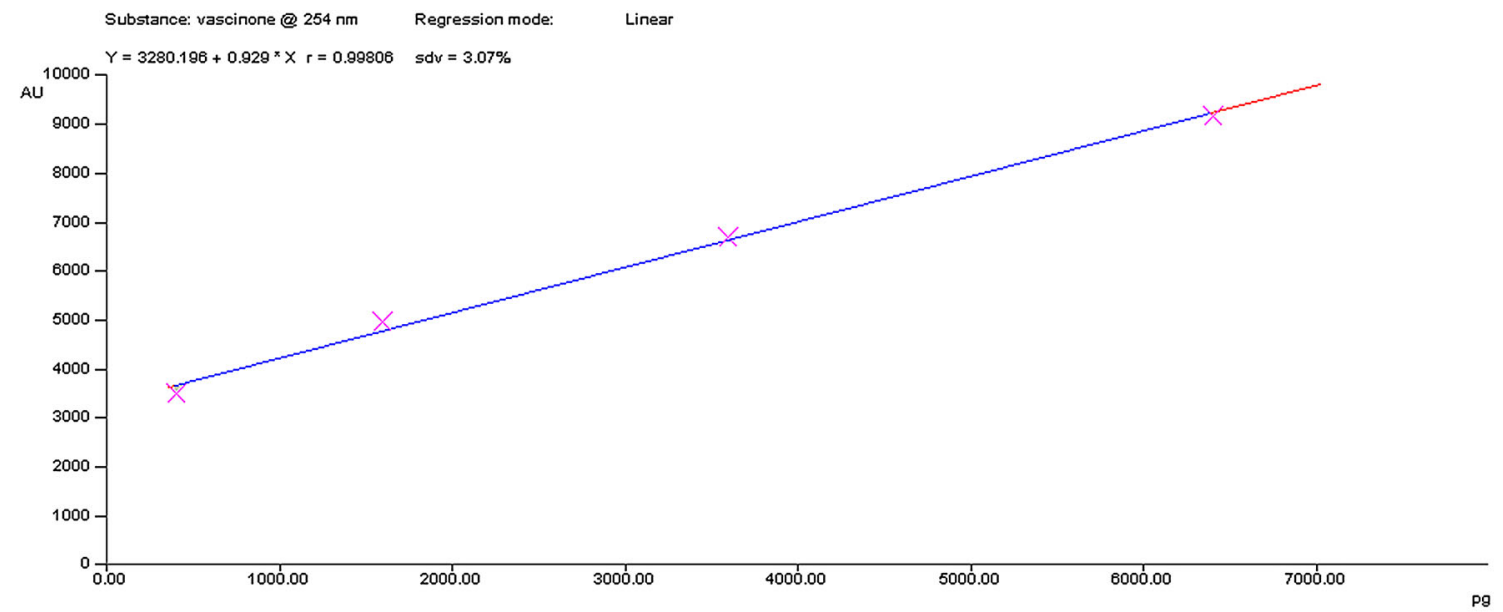

Fig. 5 Calibration curve of vasicinone 


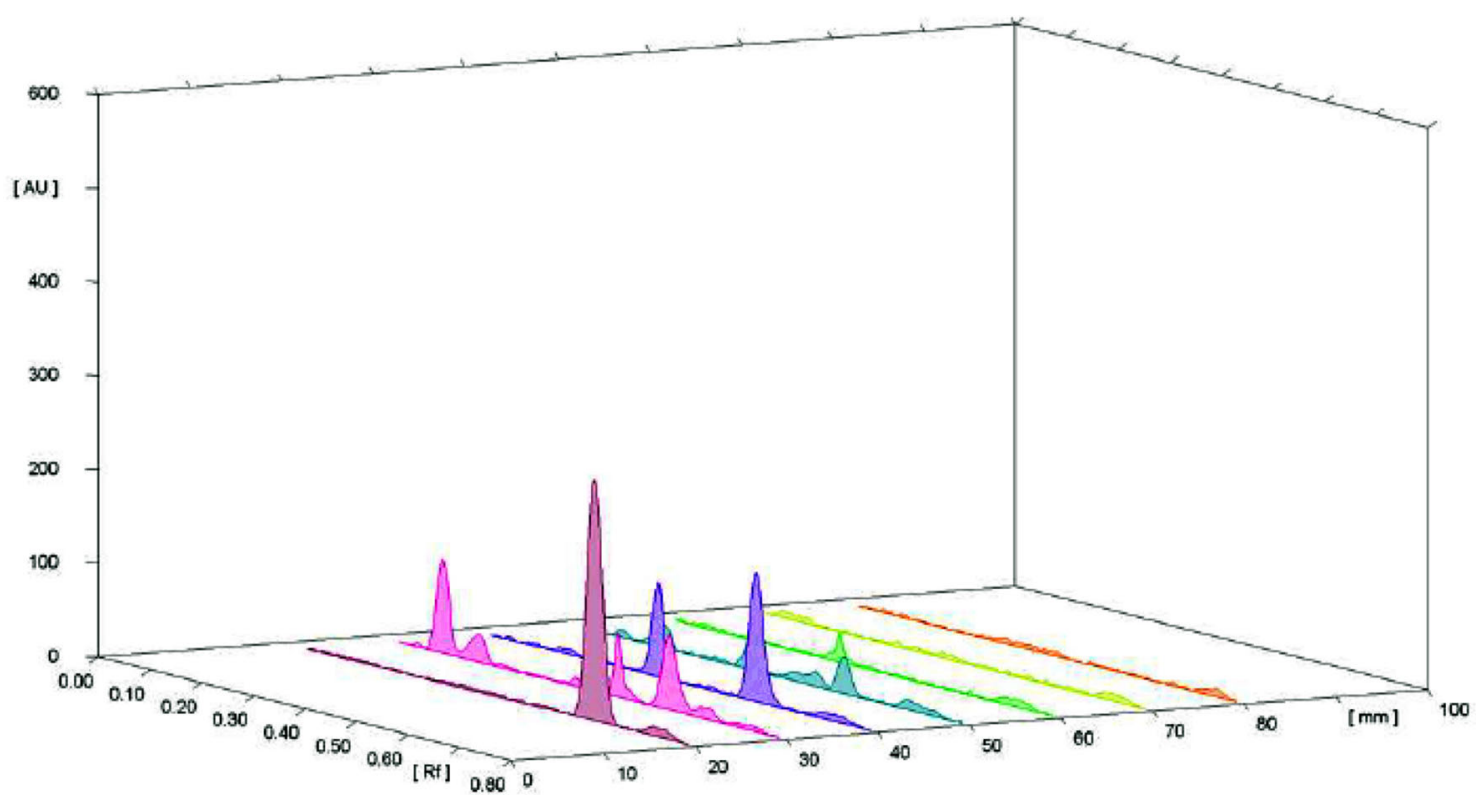

Fig. 6 Specificity in Adhatoda vasica Nees samples at $233 \mathrm{~nm}$ remission

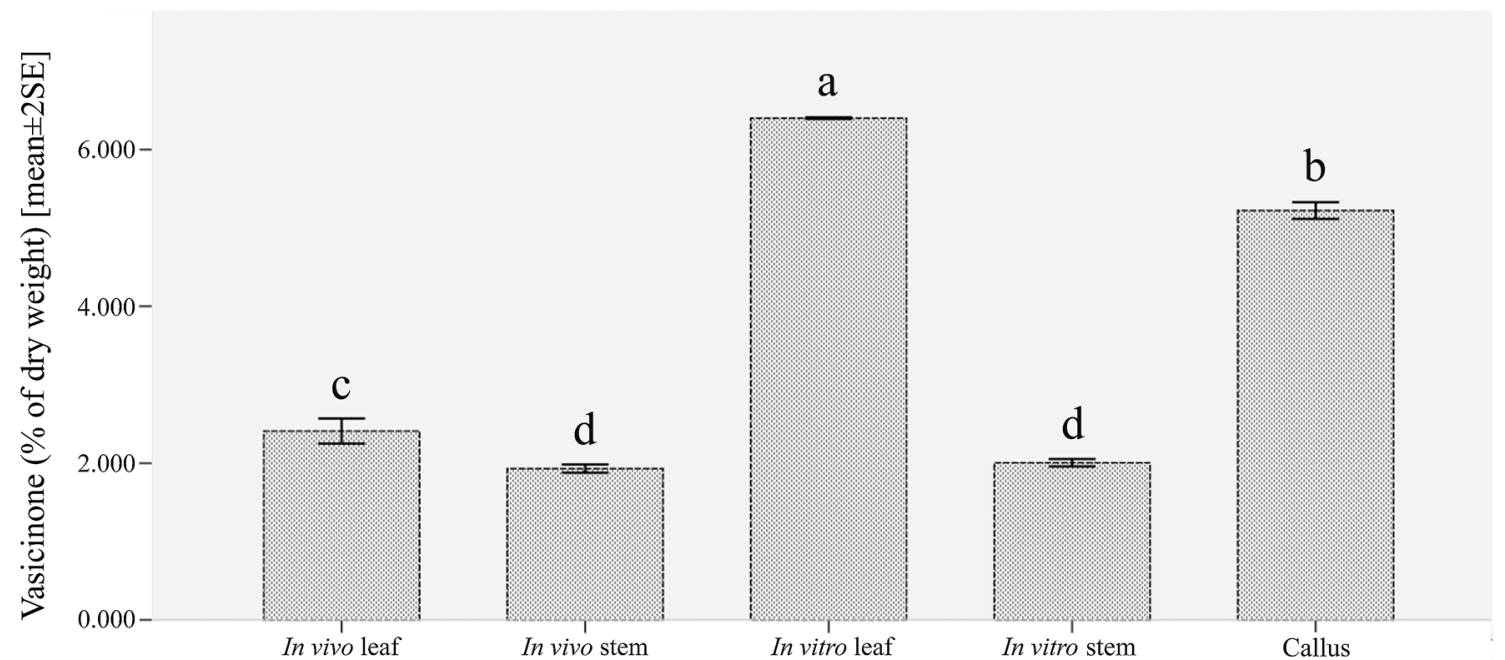

Plant parts used for vasicinone quantification

Fig. 7 Comparative vasicinone content (mean value \pm standard error bar; $n=3$ ) in various (in vivo and in vitro) plant parts of Adhatoda vasica Nees. Columns with the different letters are

environments. Such outcome certainly will encourage the pharmaceutical industry to use both these methods to complement each other to meet the bulk demand of $A$. vasica-based drug industries in the Indian subcontinent for vasicinone source. However, in a comparison, during in vivo and in vitro vasicinone production, it was detected that samples from in vitro condition produced a better yield than the other one. The highest quantity of vasicinone (6.402 $\pm 0.010 \%$ of dry weight) was measured from significant at $P=0.05$ based on one-way analysis of variance followed by Duncan's multiple range test (Duncan 1955)

in vitro leaves followed by calli $(5.222 \pm 0.092 \%)$ and in vitro stems $(2.007 \pm 0.041 \%)$. In contrast, in vivo leaves and stems produced comparatively lower levels of vasicinone $(2.412 \pm 0.139$ and $1.933 \pm 0.046 \%$, respectively) (Fig. 7). This result is corroborated by the previous report of Roja et al. (2011) who found 3-4\% vasicinone in natural plant sample and 5-6\% vasicinone in tissue cultured plant sample, when assessed through HPLC. It was noteworthy to mention that the vasicinone quantity from the in vitro 
leaves was almost 2.5-fold higher than its in vivo counterpart. An earlier study (Das et al. 2005) reported that the vasicinone production in leaves (in vivo) was higher $(0.047 \% ; w / w)$ in comparison to stem (in vivo) $(0.027 \% ; w /$ $w$ ) of $A$. vasica, which supports our results where we found leaf samples to be the superior choice for vasicinone extraction than the other plant parts. Significant variation in vasicinone content was observed among in vitro leaf, callus and in vivo leaf; nonetheless, in vitro stem and in vivo stems yielded comparable quantities of vasicinone. The coefficient of variation (CV\%) of individual samples was recorded to be $8.133 \%$ (in vivo leaf), $3.35 \%$ (in vivo stem), $0.219 \%$ (in vitro leaf), $0.129 \%$ (in vitro stem) or $2.864 \%$ (callus). The quantity of vasicinone production in our report (6.402\% from in vitro leaf) is highest among any other reports on A. vasica [(Das et al. 2005 reported $0.047 \%$ ), (Avula et al. 2008 reported 0.97\%), (Suthar et al. 2009 reported 0.45\%), (Roja et al. 2011 reported 5.2\%), (Garg et al. 2016 reported 0.56\%)]. Such augmented production of vasicinone from in vitro leaves might be attributed to the controlled physical environment of growth room and interplay among endogenous and exogenous plant growth regulators during tissue culture.

In vitro culture of plant cell, tissue and organ are accounted as an improved system to yield valuable phytochemicals with medicinal properties (Gantait and Kundu 2017). Plant growth regulators used in vitro was found to augment some metabolic activities and ameliorate phytochemical accumulation in tissue cultured plantlets unlike in their in vivo counterparts (Pandey et al. 2016). According to Gantait et al. (2014), the use of sterilizing agents, plant source, plant growth regulators, culture conditions as well as accumulation of phenolics may significantly enhance the quality of in vitro-regenerated plants. Plants are the main source to provide novel products and act as chemical models for new drug discoveries (Cox and Balick 1994). The rapid development of plant tissue culture and cell culture leads to the production of more secondary metabolites on a large scale (Ramachandra Rao and Ravishankar 2002; Debnath et al. 2006; Gantait et al. 2011). Generally, secondary plant products can be produced throughout the year with the advent of plant tissue culture. Simultaneously, the product would be reliable, predictable and independent of seasonal barriers, plus it complements the conventional production system of secondary metabolites. At times, the in vitro yield of secondary product may exceed from that of the in vivo parent plant parts (Karuppusamy 2009), similar to the present study, wherein the in vitro yield of vasicinone surpassed than that of the in vivo parts of $A$. vasica. Despite the fact that vasicinone can be harvested from any aerial plant part, our findings based on vasicinone profiling revealed that the leaf, especially the in vitro one is the key source. This way of ensuring high vasicinone yield per unit biomass, concurrently through in vivo and in vitro approach, indicates the chemical process of vasicinone to be much easier and more economical.

\section{Conclusion}

Since there is a high demand for vasicinone in the global market of plant-derived drugs, farming activities on $A$. vasica should principally be emphasized on boosting the vasicinone quantity and biomass (especially the leaves). In our present report, we proposed an avenue for large-scale production of vasicinone, simultaneously exploiting both the conventional and tissue culture methods. Furthermore, we substantiated the in vitro clonal propagation as a superior technique to yield the augmented quantity of vasicinone in comparison to in vivo propagation method; the in vitro approach can further be utilized for the amelioration of vasicinone production through biotic and abiotic elicitors and/or stresses developed during tissue culture. The implication of this work can possibly bring down the commercial cost of production of this economically important alkaloid through complementation of in vivo and in vitro approaches.

Acknowledgements Authors are grateful to Medicinal and Aromatic Research Centre, Anand Agriculture University (AAU) for providing the plant materials for the present study and Dr. M.A. Patel, Research Scientist, AAU, for his help in identifying the plant species. Authors are thankful to the ANCHROM (HPTLC specialists since 1978), Mumbai for their assistance during HPTLC analysis. Authors extend their sincere appreciation to Dr. Raktim Mukherjee, Asst. Professor in Biotechnology, Shree P.M. Patel P.G. Institute of Studies and Research in Sciences, Anand, Gujarat, for the manuscript preparation.

Authors' contribution J. Panigrahi, I.C. Patel, S. Gantait-conceived the idea of research and designed the experiments; J. Panigrahi-executed the laboratory experiments; J. Panigrahi, S. Gantait-interpreted the results and wrote the draft manuscript; J. Panigrahi, S. Gantait, I.C. Patel-scrutinized and corrected the manuscript to its submission-ready stage. All the authors approved the final version of the manuscript prior to submission.

\section{Compliance with ethical standards}

Conflict of interest The authors of this article declare that there is no conflict of interest and they do not have any financial gain from it.

Funding This research did not receive any specific grant from funding agencies in the public, commercial, or not-for-profit sectors.

Open Access This article is distributed under the terms of the Creative Commons Attribution 4.0 International License (http:// creativecommons.org/licenses/by/4.0/), which permits unrestricted use, distribution, and reproduction in any medium, provided you give appropriate credit to the original author(s) and the source, provide a link to the Creative Commons license, and indicate if changes were made.

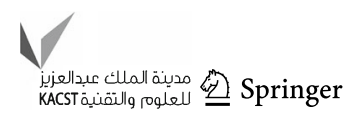




\section{References}

Ahmad S, Garg M, Ali Singh M, Attar MT, Ansari SH (2009) A phytopharmacological overview on Adhatoda zeylanica Medic, Syn. A. Vasica (Linn.) Nees. Nat Prod Rad 8:549-554

Alam P, Ali M, Singh R, Shakeel F (2011) A new HPTLC densitometric method for analysis of swertiamarin in Enicostemma littorale and commercial formulations. Nat Prod Res $25: 17-25$

Avula B, Begum S, Ahmed S, Choudhary MI, Khan IA (2008) Quantitative determination of vasicine and vasicinone in Adhatoda vasica by high performance capillary electrophoresis. Pharmazie 63:20-22

Bala M, Pratap K, Verma PK, Singh B, Padwad Y (2015) Validation of ethnomedicinal potential of Tinospora cordifolia for anticancer and immunomodulatory activities and quantification of bioactive molecules by HPTLC. J Ethnopharmacol 175:131-137

Bhide MB, Naik PY, Ghooi RB (1976) Comparative studies on the pharmacological evaluation of vasicine and vasicinone. Bull Haffkin Inst 4:43-50

Claeson UP, Malmfors T, Wikman G, Bruhn JG (2000) Adhatoda vasica: a critical review of ethnopharmacological and toxicological data. J Ethnopharmacol 72:1-20

Cox PA, Balick MJ (1994) The ethnobotanical approach to drug discovery. Sci Am 270:82-87

Das C, Poi R, Chowdhury A (2005) HPTLC determination of vasicine and vasicinone in Adhatoda vasica. Phytochem Anal 16:90-92

Debnath M, Malik CP, Bisen PS (2006) Micropropagation: a tool for the production of high quality plant-based medicines. Curr Pharm Biotechnol 7:33-49

Dharmender R, Thanki M, Agrawal R, Anandjiwala S (2010) Simultaneous quantification of bergenin, (+)-catechin, gallicin and gallic acid; and quantification of $\beta$-sitosterol using HPTLC from Bergenia ciliata (Haw.) Sternb. Forma Ligulata Yeo (Pasanbheda). Pharm Anal Acta 1:1-9

Duncan DB (1955) Multiple range and multiple F test. Biometrics 11:1-42

Gantait S, Kundu S (2017) Neoteric trends in tissue culture-mediated biotechnology of Indian ipecac [Tylophora indica (Burm. f.) Merrill]. 3Biotech 7:231

Gantait S, Mandal N, Das PK (2011) In vitro accelerated mass propagation and ex vitro evaluation of Aloe vera $\mathrm{L}$. with aloin content and superoxide dismutase activity. Nat Prod Res 25:1370-1378

Gantait S, Sinniah UR, Das PK (2014) Aloe vera: A review update on advancement of in vitro culture. Acta Agric Scand Sec B Soil Plant Sci 64:1-12

Garg M, Tamboli ET, Kamal YT, Ansari SH, Ahmad S (2016) Quantification of vasaka alkaloids in in vitro cultures and in natural leaves from Indian subcontinents by reversed phase- high performance liquid chromatography. Drug Dev Ther 7:51-54

Ignacimuthus S, Shanmugam N (2010) Antimycobacterial activity of two natural alkaloids, vasiscine acetate and 2-acetyl benzyl amine from Indian shrub Adhatoda vasica Nees. leaves. J Biosci 35:565-570

Jayapaul K, Kavi Kishor PB, Janardhan Reddy K (2005) Production of pyrroloquinazoline alkaloid from leaf and petiole-derived callus cultures of Adhatoda zeylanica. In Vitro Cell Dev Biol Plant 41:682-685

Karuppusamy S (2009) A review on trends in production of secondary metabolites from higher plants by in vitro tissue, organ and cell cultures. J Med Plant Res 3:1222-1239

Komaraiah P, Ramakrishna SV, Reddanna P, Kavi Kishor PB (2003) Enhanced production of plumbagin in immobilized cells of Plumbago rosea by elicitation and in situ adsorption. J Biotechnol 101:181-187

Kumar D, Gulati A, Sharma U (2016) Determination of theanine and catechin in Camellia sinensis (Kangra Tea) leaves by HPTLC and NMR techniques. Food Anal Methods 9:1666-1674

Mathew AS, Patel KN, Shah BK (1998) Investigation on antifeedant and anthelmintic potential of Adhatoda vasica Nees. Indian J Nat Prod 14:11-16

Murashige T, Skoog F (1962) A revised medium for rapid growth and bioassay with tobacco tissue cultures. Physiol Plant 15:473-495

Pandey DK, Parida S, Dey A (2016) Comparative HPTLC analysis of bioactive marker barbaloin from in vitro and naturally grown Aloe vera. Rev Brasil de Farmacogn 26:161-167

Panigrahi J, Patel IC (2014) Micropropagation of Adhatoda beddomei using nodal explant. Eur Acad Res II(9):12194-121204

Ramachandra Rao S, Ravishankar GA (2002) Plant cell cultures: chemical factories of secondary metabolites. Biotechnol Adv 20:101-153

Roja G, Vikrant BH, Sandur SK, Sharma A, Pushpa KK (2011) Accumulation of vasicine and vasicinone in tissue cultures of Adhatoda vasica and evaluation of the free radical-scavenging activities of the various crude extracts. Food Chem 126:1033-1038

Shinawie A (2002) Wonder drugs of medicinal plants. Ethnobotany. Mol Cell Biochem 213:99-109

Shuayprom A, Sanguansermsri D, Sanguansermsri P, Fraser IH, Wongkattiya N (2016) Quantitative determination of vitexin in Passiflora foetida Linn. leaves using HPTLC. Asian Pac J Trop Biomed 6:216-220

Singh A, Kumar S, Bajpai V, Kumar V (2016) Simultaneous determination of pyrroquinazoline alkaloids and flavonoids in Adhatoda beddomei and Adhatoda vasica and their marketed herbal formulations using ultra-high-performance liquid chromatography coupled with triple quadrupole linear ion trap mass spectrometry. Biomed Chromatogr 31:e3827

Suthar AC, Katkar KV, Patil PS, Hamarpurkar PD, Mridula G, Naik VR, Mundada GR, Chouhan VS (2009) Quantitative estimation of vasicine and vasicinone in Adhatoda vasica by HPTLC. J Pharm Res 2:1893-1899

Swain SS, Rout KK, Chand PK (2012) Production of triterpenoid anti-cancer compound taraxerol in agrobacterium-transformed root cultures of butterfly pea (Clitoria ternatea L.). Appl Biochem Biotechnol 168:487-503

Varghese S, Narmadha R, Gomathi D, Kalaiselvi M, Devaki K (2013) Phytochemical screening and HPTLC finger printing analysis of Citrullus lanatus (Thunb.) seed. J Acute Dis 2:122-126

World Health Organization (1990) The use of traditional medicine in primary health care. A manual for health workers in South-East Asia, SEARO Regional Health Papers, No. 19, New Delhi, India, pp 1-2 